\title{
UNIVERSALITY OF RANDOM GRAPHS FOR GRAPHS OF MAXIMUM DEGREE TWO
}

\author{
JEONG HAN KIM AND SANG JUNE LEE
}

\begin{abstract}
For a family $\mathcal{F}$ of graphs, a graph $G$ is called $\mathcal{F}$-universal if $G$ contains every graph in $\mathcal{F}$ as a subgraph. Let $\mathcal{F}_{n}(d)$ be the family of all graphs on $n$ vertices with maximum degree at most $d$. Dellamonica, Kohayakawa, Rödl and Ruciński [17] showed that, for $d \geq 3$, the random graph $G(n, p)$ is $\mathcal{F}_{n}(d)$-universal with high probability provided $p \geq C\left(\frac{\log n}{n}\right)^{1 / d}$ for a sufficiently large constant $C=C(d)$. In this paper we prove the missing part of the result, that is, the random graph $G(n, p)$ is $\mathcal{F}_{n}(2)$-universal with high probability provided $p \geq C\left(\frac{\log n}{n}\right)^{1 / 2}$ for a sufficiently large constant $C$.
\end{abstract}

\section{INTRODUCTION}

For a positive integer $n$ and a real number $p$ in the range $0 \leq p \leq 1$, the random graph $G(n, p)$ on a set $V$ of $n$ elements may be obtained from the complete graph on $V$ by choosing each edge with probability $p$, independently of all other edges.

After the random graph $G(n, p)$ was first introduced by Erdös 22] in 1947, the theory of the random graph has become an active area of research. One of the most interesting problems is the containment problem, in which one tries to obtain conditions on $p$ for the property that $G(n, p)$ contains a given graph $H$ as a subgraph with high probability. For example, when $n$ is even and the given graph $H$ is a perfect matching on $V$, then it is easy to see that $n p-\log n \rightarrow \infty$ is a necessary condition, as there is an isolated vertex with substantial probability if $n p-\log n$ is bounded. Erdős and Rényi [23] showed the condition is also sufficient. In the case that $H$ is a Hamiltonian cycle, Komlós and Szemerédi [29] and Korshunov [30] discovered that the easy necessary condition $n p-\log n-\log \log n \rightarrow \infty$ is also sufficient. More generally, if $H$ is a factor of a strictly balanced graph, including a triangle, a cycle or a complete graph, Johansson, Kahn and $\mathrm{Vu}$ [28] determined a necessary and sufficient condition for the containment problem with respect to $H$. For more information, the reader is referred to Bollobás [11] and Janson, Euczak and Ruciński [26] and the references therein.

One may also consider a family $\mathcal{F}$ of graphs rather than a single graph $H$. For a family $\mathcal{F}$ of graphs, a graph $G$ is called $\mathcal{F}$-universal if $G$ contains every graph in $\mathcal{F}$ as a subgraph. There is extensive research on $\mathcal{F}$-universal graphs when $\mathcal{F}$ are families of trees [13, 16], spanning trees [10,

Date: October 22, 2013.

The first author was supported by the National Research Foundation of Korea (NRF) Grant funded by the Korean Government (MSIP) (NRF-2012R1A2A2A01018585) and KIAS internal Research Fund CG046001.

The second author was supported by the National Research Foundation of Korea (NRF) Grant funded by the Korea Government (MSIP)(No. 2013042157). This work was partially done while the second author was visiting Korea Institute for Advanced Study (KIAS). 
[14, 15, 24, planar graphs of bounded degree [10], graphs of bounded size [7, 32, graphs of bounded degree [2, 3, 4, 5, 12], and spanning graphs of bounded degree [1, 27], etc.

Since an $\mathcal{F}$-universal graph $G$ must have the maximum degree greater than or equal to the maximum degrees of all graphs in $\mathcal{F}$, a family $\mathcal{F}$ of graphs of bounded degree may be considered. For example, one may consider the family $\mathcal{T}_{n}(d)$ of all trees on $n$ vertices with maximum degree at most d. Bhatt, Chung, Leighton and Rosenberg [10] showed that there is a $\mathcal{T}_{n}(d)$-universal graph on $n$ vertices with maximum degree depending only on $d$. For $d \geq \log n$, Johannsen, Krivelevich and Samotij [27] proved that there is a positive constant $c$ such that $G(n, p)$ with $p \geq c d n^{-1 / 3} \log n$ is asymptotically almost surely (a.a.s.) $\mathcal{T}_{n}(d)$-universal; where, in general, a property holds asymptotically almost surely, or simply a.a.s., if it holds with probability tending to 1 as $n \rightarrow \infty$. In particular, we have that $G(n, p)$ with $p \geq c n^{-1 / 3}(\log n)^{2}$ is a.a.s. $\mathcal{T}_{n}(\log n)$-universal, and hence, $\mathcal{T}_{n}(d)$-universal for a constant $d$. For the family $\mathcal{T}_{(1-\varepsilon) n}(d)$ of all trees on $(1-\varepsilon) n$ vertices with maximum degree at most $d$, Alon, Krivelevich and Sudakov [6] showed that for every positive constant $\varepsilon$ and positive integer $d$, there exists a constant $c=c(\varepsilon, d)$ such that $G(n, p)$ with $p=c / n$ is a.a.s. $\mathcal{T}_{(1-\varepsilon) n}(d)$-universal. For more related results, [19, 8, 9] may be referred.

In this paper, we consider the family $\mathcal{F}_{n}(d)$ of all graphs on $n$ vertices with maximum degree at most $d$. For an even $n$ and $d=1$, the $\mathcal{F}_{n}(1)$-universality is equivalent to the containment problem for a perfect matching. Provided that $n$ is divisible by $d+1$, one may easily see that $p \geq\left(\frac{(\log n)^{1 / d}}{n}\right)^{2 /(d+1)}$ is a necessary condition for $G(n, p)$ being a.a.s. $\mathcal{F}_{n}(d)$-universal, since $\mathcal{F}_{n}(d)$ contains a $K_{d+1}$-factor, and hence, every vertex must be contained in a copy of $K_{d+1}$. On the other hand, Dellamonica, Kohayakawa, Rödl and Ruciński [20, 21] proved that $p \geq C\left(\frac{\log n}{n}\right)^{1 /(2 d)}$ is sufficient, for a sufficiently large constant $C$.

Theorem 1 ([20, 21]). For every integer $d \geq 2$, there exists a positive constant $C=C(d)$ such that if $p \geq C\left(\frac{(\log n)^{2}}{n}\right)^{1 /(2 d)}$, then the random graph $G(n, p)$ is a.a.s. $\mathcal{F}_{n}(d)$-universal.

Recently the above result was notably improved for $d \geq 3$.

Theorem 2 ([17, 18]). For every integer $d \geq 3$, there exists a positive constant $C=C(d)$ such that if $p \geq C\left(\frac{\log n}{n}\right)^{1 / d}$, then the random graph $G(n, p)$ is a.a.s. $\mathcal{F}_{n}(d)$-universal.

In this paper, we show that the statement of Theorem 2 holds for $d=2$.

Theorem 3. There exists a positive constant $C$ such that if $p \geq C\left(\frac{\log n}{n}\right)^{1 / 2}$, then the random graph $G(n, p)$ is a.a.s. $\mathcal{F}_{n}(2)$-universal.

The rest of this paper is organized as follows. In the next section we define a notion of a 'good' graph and introduce two main lemmas, which imply Theorem 3 . Sections 3 and 4 are for the proofs of the two lemmas.

In this paper, we will use the following notation and convention.

Notation and convention: For a graph $G$ and $v \in V(G)$, the set $N_{G}(v)$ denotes the set of neighbors of $v$ in $G$. Similarly, for $U \subset V(G)$, the set $N_{G}(U)$ denotes the set of vertices which are 
adjacent to a vertex in $U$. The graph $G[U]$ denotes the subgraph of $G$ induced on $U$. For simplicity, we omit floor and ceiling symbols when they are not essential.

\section{GoOd GRAPH AND TWO LEMMAS}

In order to show Theorem 3 , by monotonicity, it suffices to show the statement of Theorem 3 with $p=C\left(\frac{\log n}{n}\right)^{1 / 2}$ for a sufficiently large constant $C$. Hence, from now on, we fix $p$ as $p=C\left(\frac{\log n}{n}\right)^{1 / 2}$. Throughout the paper, we let

$$
\delta=0.01 \quad \text { and } \quad \varepsilon=0.001
$$

Now we provide the definition of a 'good' graph. Let $V$ be a vertex set on $n$ vertices. We fix a partition $V=V_{0} \cup V_{1} \cup \cdots \cup V_{6}$ such that

$$
\left|V_{1}\right|=\cdots=\left|V_{6}\right|=\varepsilon n \quad \text { and } \quad\left|V_{0}\right|=(1-6 \varepsilon) n \geq(3 / 4) n .
$$

For a graph $G$ on $V$ and $k=1$ or 2 , let $U \subset V$ and $\mathcal{L}$ be a collection of pairwise disjoint $k$-subsets of $V \backslash U$. We consider a bipartite graph $B(\mathcal{L}, U)$ between $\mathcal{L}$ and $U$, in which $L \in \mathcal{L}$ and $u \in U$ are adjacent if and only if $L \subset N_{G}(u)$.

Now we are ready to define a good graph.

Definition 4. A graph $G$ on $V$ is called ' $(n, C)$-good' if the following properties hold.

$(P 1)$ There exists a matching $\mathcal{M}$ of $G\left[V_{0}\right]$ with $|\mathcal{M}|=2 \varepsilon n$ such that for all $U \subset V \backslash V(\mathcal{M})$ with $|U| \leq \frac{\delta n}{C^{2} \log n}$, we have

$$
\mid\{\{a, b\} \in \mathcal{M} \mid a \sim u, b \sim u \text { for some } u \in U\}\left|\geq \frac{C^{2} \log n}{16 n}\right| \mathcal{M}|| U \mid .
$$

$(P 2)$ Let $k=1$ or 2 , and $\mathcal{L}$ be a collection of pairwise disjoint $k$-subsets of $V$.

$$
\begin{aligned}
& \text { If }|\mathcal{L}| \leq \frac{\delta}{C^{k}}\left(\frac{n}{\log n}\right)^{k / 2}, \text { then, for } V_{i} \text { with } V_{i} \cap\left(\bigcup_{L \in \mathcal{L}} L\right)=\emptyset, i=1, \ldots, 6 \text {, we have that } \\
& \qquad\left|N_{B\left(\mathcal{L}, V_{i}\right)}(\mathcal{L})\right| \geq(1-\delta) C^{k}\left(\frac{\log n}{n}\right)^{k / 2}|\mathcal{L}|\left|V_{i}\right| . \\
& \text { If }|\mathcal{L}| \geq \frac{\log n}{C^{k-1}}\left(\frac{n}{\log n}\right)^{k / 2}, \text { then, for all } U \text { with }|U| \geq \frac{\log n}{C^{k-1}}\left(\frac{n}{\log n}\right)^{k / 2} \text { and } U \cap\left(\bigcup_{L \in \mathcal{L}} L\right)=
\end{aligned}
$$

$\emptyset$, the graph $B(\mathcal{L}, U)$ has at least one edge.

$$
\left(\text { No requirement is needed when } \frac{\delta}{C^{k}}\left(\frac{n}{\log n}\right)^{k / 2}<|\mathcal{L}|<\frac{\log n}{C^{k-1}}\left(\frac{n}{\log n}\right)^{k / 2}\right) \text {. }
$$

Remark 5. For $p=C\left(\frac{\log n}{n}\right)^{1 / 2}$, the above inequality (2.1) may be written as

$$
\left|N_{B\left(\mathcal{L}, V_{i}\right)}(\mathcal{L})\right| \geq(1-\delta) p^{k}|\mathcal{L}|\left|V_{i}\right|
$$

Notice that $p^{k}|\mathcal{L}|\left|V_{i}\right|$ is the expected number of edges in $B\left(\mathcal{L}, V_{i}\right)$ if $G$ were $G(n, p)$. It is easy to see that only few vertices of $V_{i}$ are of degree 2 or more in $B\left(\mathcal{L}, V_{i}\right)$. Hence, $\left|N_{B\left(\mathcal{L}, V_{i}\right)}(\mathcal{L})\right|$ is almost the same as the number of edges in $B\left(\mathcal{L}, V_{i}\right)$.

We will show the following two lemmas. 
Lemma 6. There exists a positive constant $C$ such that an $(n, C)$-good graph is $\mathcal{F}_{n}(2)$-universal provided that $n$ is sufficiently large.

Lemma 7. There exists a positive constant $C$ such that the random graph $G(n, p)$ on $V$ with $p=C\left(\frac{\log n}{n}\right)^{1 / 2}$ is a.a.s. $(n, C)$-good.

Our proof of Lemmas 6 and 7 will be given in Sections 3 and 4 , respectively. Theorem 3 clearly follows from Lemmas 6 and 7 .

\section{UNIVERSALITY OF GOOD GRAPH}

For the proof of Lemma 6, we may assume that $H$ is a maximal graph in $\mathcal{F}_{n}(2)$, in the sense that no edge may be added to $H$ to be a graph in $\mathcal{F}_{n}(2)$. Then, it is easy to see that all but at most one vertex of $H$ have degree 2 . We will show that there exists a positive constant $C$ such that, for a sufficiently large $n$, an $(n, C)$-good graph $G$ contains a copy of $H$ as a subgraph. To this end, a partition of $W:=V(H)$ will be used, and each part will be embedded at a time. A subset of $W$ is called $k$-independent in $H$ if the distance between every distinct pair of vertices in the subset is greater than $k$.

Lemma 8. Let $H$ be a maximal graph in $\mathcal{F}_{n}(2)$. Then there is a partition $W:=V(H)=W_{0} \cup$ $W_{1} \cup \cdots \cup W_{6}$ with

$$
\left|W_{0}\right|=4 \varepsilon n, \quad\left|W_{6}\right|=2 \varepsilon n, \quad\left|W_{i}\right| \geq 2 \varepsilon n, \quad i=1,2, \ldots, 5,
$$

such that

(1) $W_{1}, \ldots, W_{5}$ are 2 -independent.

(2) $W_{6}$ is 3-independent, and all vertices of $W_{6}$ are of degree 2.

(3) $W_{0}=N_{H}\left(W_{6}\right)$.

Proof. We first construct $W_{6}$ and $W_{0}$. Since the maximum degree of $H$ is 2, for each vertex $v$ in $H$, there are at most 6 vertices that are of distance 3 or less from $v$, excluding $v$ itself. By the greedy algorithm, it is easy to see that there is a 3 -independent set of size at least $n / 7$. Hence, we may choose $W_{6}$ satisfying $\left|W_{6}\right|=2 \varepsilon n$ and (2) as there is at most one vertex of degree less than 2. Let $W_{0}:=\bigcup_{w \in V_{6}} N_{H}(w)$. Clearly, $\left|W_{0}\right|=4 \varepsilon n$ as $W_{6}$ is 3-independent.

Next, we consider $W_{i}$ for $1 \leq i \leq 5$. Let $H^{2}$ be the graph on the vertex set $W$ in which two vertices are adjacent if and only if two vertices are of distance at most 2 in $H$. Since $H$ has the maximum degree 2, the maximum degree of $H^{2}$ is at most 4. Using Hajnal-Szemerédi Theorem [25], we may partition $W$ into 5 independent sets of $H^{2}$ so that each part is of size at least $n / 5-1$. By removing all vertices in $W_{0} \cup W_{6}$ from each part, $W_{1}, W_{2}, \ldots, W_{5}$ may be obtained. Then, it is clear that each $W_{i}$ is 2 -independent in $H$ and $\left|W_{i}\right|$ is at least $n / 5-1-6 \varepsilon n \geq 2 \varepsilon n$, for $i=1, \ldots, 5$.

A bijection from $W$ to $V=V(G)$ is called an embedding of $H$ to $G$ if it maps each edge of $H$ to an edge of $G$. We now find an embedding of $H$ to $G$ using an algorithm modified from the embedding algorithm in [17, 18]: Take a partition $W_{0}, \ldots, W_{6}$ of $W$ as described in Lemma 8. We will embed $W_{i}$ into $V_{0} \cup \cdots \cup V_{i}$. 


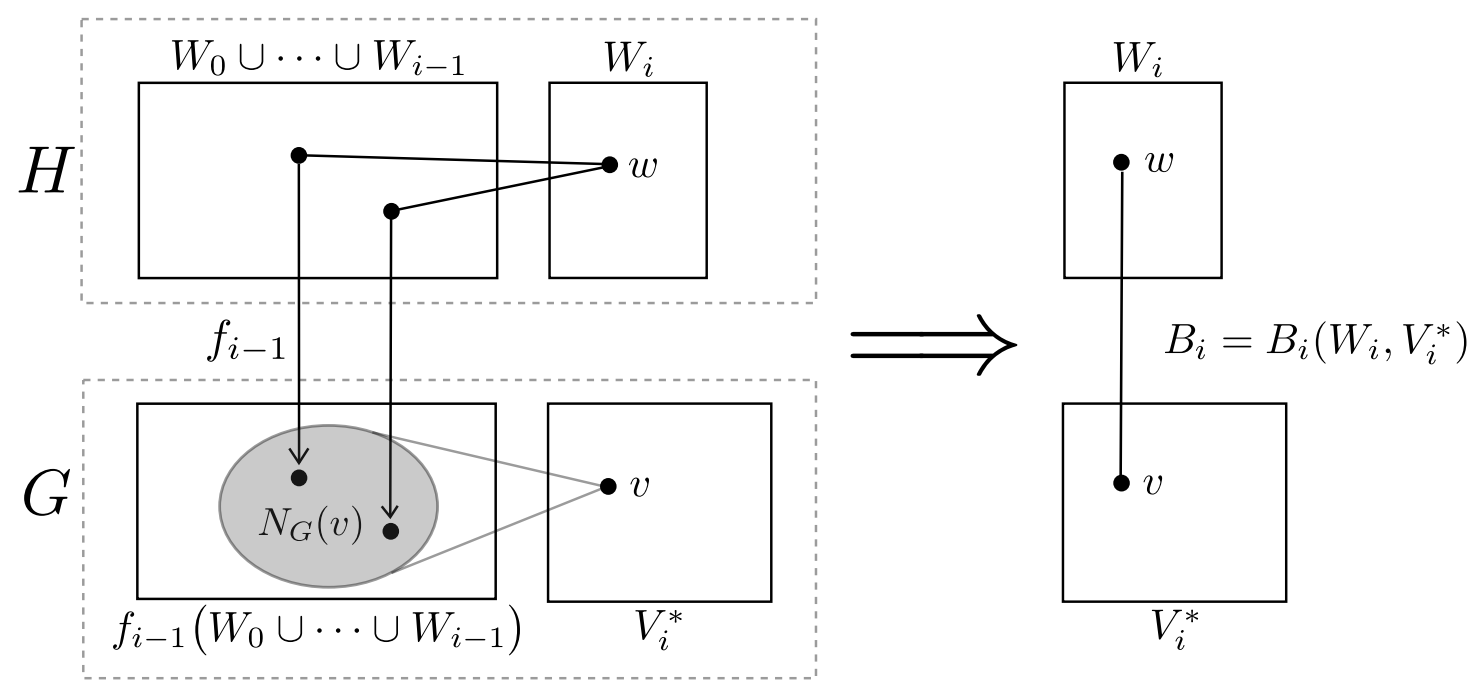

Figure 1. The bipartite graph $B_{i}=B_{i}\left(W_{i}, V_{i}^{*}\right)$

To map $W_{0}$ into $V_{0}$, recall that $W_{0}=N_{H}\left(W_{6}\right)$ and $\left|N_{H}(w)\right|=2$ for all $w \in W_{6}$. For a matching $\mathcal{M}=\left\{e_{1}, \ldots, e_{2 \varepsilon n}\right\}$ in $G\left[V_{0}\right]$ with $(\mathrm{P} 1)$ and $W_{6}=\left\{w_{i}, \ldots, w_{2 \varepsilon n}\right\}$, it is enough for us to take a bijection, say $f_{0}$, from $W_{0}$ to $V(\mathcal{M})$ such that $N_{H}\left(w_{i}\right)$ is mapped to $e_{i}$, where $V(\mathcal{M})$ is the set of end vertices of all edges in $\mathcal{M}$.

The mapping $f_{0}$ is an embedding of $W_{0}$ to $V_{0}$ : Since $V_{6}$ is a 3 -independent set in $H$, the sets $N_{H}(w), w \in V_{6}$, are pairwise disjoint and there is no edge between them. Hence, every edge $e$ in $W_{0}$ belongs to $N_{H}(w)$ for some $w \in W_{6}$. As every $N_{H}(w)$ is mapped to an edge in $\mathcal{M} \subset E\left(G\left[V_{0}\right]\right)$ under $f_{0}$, the edge $e$ is mapped to an edge in $G\left[V_{0}\right]$.

Assuming an embedding

$$
f_{i-1}: W_{0} \cup W_{1} \cup \cdots \cup W_{i-1} \rightarrow V_{0} \cup V_{1} \cup \cdots \cup V_{i-1}
$$

is defined, $i=1,2, \ldots, 6$, we will embed $W_{i}$ into $V_{i}^{*}:=V_{i} \cup\left(V_{0} \cup V_{1} \cup \cdots \cup V_{i-1}\right) \backslash \operatorname{Image}\left(f_{i-1}\right)$ to extend $f_{i-1}$ to an embedding

$$
f_{i}: W_{0} \cup W_{1} \cup \cdots \cup W_{i} \rightarrow V_{0} \cup V_{1} \cup \cdots \cup V_{i} .
$$

Let $B_{i}\left(W_{i}, V_{i}^{*}\right)$, or just $B_{i}$, be the bipartite graph in which $w \in W_{i}$ and $v \in V_{i}^{*}$ are adjacent if and only if

$$
f_{i-1}\left(N_{H}(w) \cap\left(W_{0} \cup \cdots \cup W_{i-1}\right)\right) \subset N_{G}(v) .
$$

(See Figure 11. Or equivalently, for $L_{i}(w):=f_{i-1}\left(N_{H}(w) \cap\left(W_{0} \cup \cdots \cup W_{i-1}\right)\right), \mathcal{L}_{i}:=\left\{L_{i}(w)\right.$ : $\left.w \in W_{i}\right\}$ and the bipartite graph $B\left(\mathcal{L}_{i}, V_{i}^{*}\right)$ defined just before Definition 4

$$
w \sim v \text { in } B_{i}\left(W_{i}, V_{i}^{*}\right) \text { if and only if } L_{i}(w) \sim v \text { in } B\left(\mathcal{L}_{i}, V_{i}^{*}\right) .
$$

If possible, take a $W_{i}$-matching of $B_{i}$, i.e., a matching in $B_{i}$ that covers all vertices in $W_{i}$. (Later, we will show that this is possible). The image of $w \in W_{i}$ under the mapping $f_{i}$ is defined to be the vertex in $V_{i}^{*}$ that is matched to $w$ in the $W_{i}$-matching. For $w \notin W_{i}, f_{i}(w)=f_{i-1}(w)$. 
The mapping $f_{i}$ is an embedding of $W_{i}$ to $V_{i}^{*}$ : For an edge $e$ in $W_{0} \cup \cdots \cup W_{i}$, at most one end point of $e$ is in $W_{i}$ since $W_{i}$ is 2-independent, especially independent. If both ends of $e$ are in $W_{0} \cup \cdots \cup W_{i-1}$, then $f_{i}(e)=f_{i-1}(e)$ is an edge in $G$. If one end, say $w$, of $e$ is in $W_{i}$, then the other end, say $w^{\prime}$, is in $N_{H}(w) \cap\left(W_{0} \cup \cdots \cup W_{i-1}\right)$. Hence, $w \sim f_{i}(w)$ in $B_{i}\left(W_{i}, V_{i}^{*}\right)$ implies that

$$
f_{i-1}\left(N_{H}(w) \cap\left(W_{0} \cup \cdots \cup W_{i-1}\right)\right) \subset N_{G}\left(f_{i}(w)\right),
$$

in particular, $f_{i}\left(w^{\prime}\right)=f_{i-1}\left(w^{\prime}\right) \in N_{G}\left(f_{i}(w)\right)$, i.e., $\left\{f_{i}\left(w^{\prime}\right), f_{i}(w)\right\}$ is an edge.

It remains to show that there exists a $W_{i}$-matching in $B_{i}=B_{i}\left(W_{i}, V_{i}^{*}\right)$ for $i=1, \ldots, 6$. We first show the following, which guarantees Hall's condition for a subset $U$ of $W_{i}$ satisfying some condition.

Lemma 9. Let $i=1, \ldots, 6$. If $U \subset W_{i}$ satisfying $|U| \leq\left|V_{i}^{*}\right|-n / C$, then

$$
\left|N_{B_{i}}(U)\right| \geq|U| \text {. }
$$

Proof. Let $U=U_{0} \cup U_{1} \cup U_{2}$, where

$$
U_{j}:=\left\{w \in W_{i}:\left|N_{H}(w) \cap\left(W_{0} \cup \cdots \cup W_{i-1}\right)\right|=j\right\} .
$$

If $U_{0} \neq \emptyset$, then $N_{B_{i}}(U)=V_{i}^{*}$ and hence $\left|N_{B_{i}}(U)\right|=\left|V_{i}^{*}\right|>|U|$ as $|U| \leq\left|V_{i}^{*}\right|-n / C$. We now assume that $U_{0}=\emptyset$. Take $U_{k}$ such that $\left|U_{k}\right| \geq|U| / 2$.

Case 1: the case when $\left|U_{k}\right| \leq \frac{\delta}{C^{k}}\left(\frac{n}{\log n}\right)^{k / 2}$. For $L_{i}(u):=f_{i-1}\left(N_{H}(u) \cap\left(W_{1} \cup \cdots \cup W_{i-1}\right)\right)$ and $\mathcal{L}\left(U_{k}\right)=\left\{L_{i}(u): u \in U_{k}\right\}$, We have that

$$
N_{B\left(\mathcal{L}_{i}, V_{i}^{*}\right)}\left(\mathcal{L}\left(U_{k}\right)\right) \cap V_{i} \subset N_{B_{i}}\left(U_{k}\right):
$$

For $v \in V_{i}$ with $L(u) \sim v$ in $B\left(\mathcal{L}_{i}, V_{i}^{*}\right)$ for some $u \in U_{k}$, it follows from (3.2) that $u \sim v$ in $B_{i}$ for $u \in U_{k}$, or equivalently, $v \in N_{B_{i}}\left(U_{k}\right)$. Notice that

$$
N_{B\left(\mathcal{L}_{i}, V_{i}^{*}\right)}\left(\mathcal{L}\left(U_{k}\right)\right) \cap V_{i}=N_{B\left(\mathcal{L}\left(U_{k}\right), V_{i}\right)}\left(\mathcal{L}\left(U_{k}\right)\right) .
$$

Property (P2) implies that

$$
\begin{aligned}
\left|N_{B_{i}}\left(U_{k}\right)\right| & \geq\left|N_{B\left(\mathcal{L}\left(U_{k}\right), V_{i}\right)}\left(\mathcal{L}\left(U_{k}\right)\right)\right| \geq(1-\delta) C^{k} \varepsilon n\left(\frac{\log n}{n}\right)^{k / 2}\left|U_{k}\right| \\
& \geq \frac{\varepsilon C^{2}(\log n)}{2}\left|U_{k}\right|,
\end{aligned}
$$

as $k=1$ or 2 . In particular, $\left|N_{B_{i}}\left(U_{k}\right)\right| \geq 2\left|U_{k}\right| \geq|U|$.

Case 2: the case when $\frac{\delta}{C^{k}}\left(\frac{n}{\log n}\right)^{k / 2}<\left|U_{k}\right| \leq \frac{\log n}{C^{k-1}}\left(\frac{n}{\log n}\right)^{k / 2}$. Taking a subset $U_{k}^{\prime}$ of $U_{k}$ of size $\frac{\delta}{C^{k}}\left(\frac{n}{\log n}\right)^{k / 2}$, it follows from $(3.3)$ that

$$
\begin{aligned}
\left|N_{B_{i}}\left(U_{k}\right)\right| & \geq\left|N_{B_{i}}\left(U_{k}^{\prime}\right)\right| \geq \frac{\varepsilon \delta C^{2-k}(\log n)}{2}\left(\frac{n}{\log n}\right)^{k / 2} \\
& =\frac{\varepsilon \delta C}{2} \cdot \frac{\log n}{C^{k-1}}\left(\frac{n}{\log n}\right)^{k / 2} \geq 2\left|U_{k}\right| \geq|U|
\end{aligned}
$$


as $C$ is sufficiently large and $\varepsilon$ and $\delta$ are absolute constants.

Case 3: the case when $\left|U_{k}\right|>\frac{\log n}{C^{k-1}}\left(\frac{n}{\log n}\right)^{k / 2}$. We will show that

$$
\left|N_{B_{i}}\left(U_{k}\right)\right| \geq\left|V_{i}^{*}\right|-n / C(\geq|U|) .
$$

We first observe that there is no edge of $B_{i}=B_{i}\left(W_{i}, V_{i}^{*}\right)$ between $U_{k}$ and $V_{i}^{*} \backslash N_{B_{i}}\left(U_{k}\right)$. Hence, for $\mathcal{L}\left(U_{k}\right)$ defined as in Case 1, it follows from 3.2 that there is no edge of $B\left(\mathcal{L}_{i}, V_{i}^{*}\right)$ between $\mathcal{L}\left(U_{k}\right)$ and $V_{i}^{*} \backslash N_{B_{i}}\left(U_{k}\right)$. This means that the induced subgraph $B\left(\mathcal{L}\left(U_{k}\right), V_{i}^{*} \backslash N_{B_{i}}\left(U_{k}\right)\right)$ of $B\left(\mathcal{L}_{i}, V_{i}^{*}\right)$ has no edge. Since $\left|\mathcal{L}\left(U_{k}\right)\right|=\left|U_{k}\right|>\frac{\log n}{C^{k-1}}\left(\frac{n}{\log n}\right)^{k / 2}$, the property (P2) yields that

$$
\left|V_{i}^{*} \backslash N_{B_{i}}\left(U_{k}\right)\right|<\frac{\log n}{C^{k-1}}\left(\frac{n}{\log n}\right)^{k / 2} \leq \frac{n}{C},
$$

which is equivalent to $\left|N_{B_{i}}\left(U_{k}\right)\right| \geq\left|V_{i}^{*}\right|-n / C$ as desired.

Corollary 10. For $i=1, \ldots, 5$, there exists a $W_{i}$-matching in $B_{i}\left(W_{i}, V_{i}^{*}\right)$.

Proof. One can easily show that $\left|W_{i}\right|<\left|V_{i}^{*}\right|-n / C$ for $i=1, \ldots, 5$. Indeed, we have

$$
\begin{aligned}
\left|V_{i}^{*}\right| & =\left|V_{0} \cup \cdots \cup V_{i}\right|-\left|W_{0} \cup \cdots \cup W_{i-1}\right| \\
& =\left|W_{i} \cup \cdots \cup W_{6}\right|-\left|V_{i+1} \cup \cdots \cup V_{6}\right|,
\end{aligned}
$$

and

$$
\begin{aligned}
\left|V_{i}^{*}\right|-\left|W_{i}\right| & =\left|W_{i+1} \cup \cdots \cup W_{6}\right|-\left|V_{i+1} \cup \cdots \cup V_{6}\right| \\
& \geq(6-i) 2 \varepsilon n-(6-i) \varepsilon n=(6-i) \varepsilon n \geq \varepsilon n>\frac{n}{C}
\end{aligned}
$$

where the first inequality follows from (3.1) and the last inequality holds for a sufficiently large constant $C$. Clearly, for all $U \subset W_{i}$, we have $|U|<\left|V_{i}^{*}\right|-n / C$ for $1 \leq i \leq 5$. Hence, Lemma 9 yields that for every $U \subset W_{i}$, we have $\left|N_{B_{i}}(U)\right| \geq|U|$. Consequently, Hall's theorem implies Corollary 10.

Next, we consider the case when $i=6$.

Lemma 11. There exists a $W_{6}$-matching in $B_{6}=B_{6}\left(W_{6}, V_{6}^{*}\right)$.

Proof. It suffices to check Hall's condition, that is, for every $U \subset W_{6}$,

$$
\left|N_{B_{6}}(U)\right| \geq|U| \text {. }
$$

If $|U| \leq\left|V_{6}^{*}\right|-n / C=2 \varepsilon n-n / C$, then Lemma 9 implies (3.4). Hence, we assume that

$$
|U|>2 \varepsilon n-n / C \text {. }
$$

Notice that

$$
|U|+\left|N_{B_{6}}\left(V_{6}^{*} \backslash N_{B_{6}}(U)\right)\right| \leq\left|W_{6}\right|=2 \varepsilon n
$$


since $U$ and $N_{B_{6}}\left(V_{6}^{*} \backslash N_{B_{6}}(U)\right)$ are disjoint. If $\left|V_{6}^{*} \backslash N_{B_{6}}(U)\right| \geq \frac{\delta n}{C^{2} \log n}$, take a subset $Y$ of $V_{6}^{*} \backslash N_{B_{6}}(U)$ with $|Y|=\frac{\delta n}{C^{2} \log n}$. Then, by equation (3.2) and Property (P1), we infer

$$
\left|N_{B_{6}}\left(V_{6}^{*} \backslash N_{B_{6}}(U)\right)\right| \geq\left|N_{B_{6}}(Y)\right| \geq \frac{\varepsilon \delta}{8} n>\frac{n}{C}
$$

and $|U|+\left|N_{B_{6}}\left(V_{6}^{*} \backslash N_{B_{6}}(U)\right)\right|>2 \varepsilon n$, which is a contradiction. Therefore, $\left|V_{6}^{*} \backslash N_{B_{6}}(U)\right|<\frac{\delta n}{C^{2} \log n}$. Then, Property (P1) together with 3.2 implies that

$$
\left|N_{B_{6}}\left(V_{6}^{*} \backslash N_{B_{6}}(U)\right)\right| \geq \frac{C^{2} \varepsilon \log n}{8}\left|V_{6}^{*} \backslash N_{B_{6}}(U)\right|>\left|V_{6}^{*} \backslash N_{B_{6}}(U)\right| .
$$

Since $\left|N_{B_{6}}(U)\right|+\left|V_{6}^{*} \backslash N_{B_{6}}(U)\right|=\left|V_{6}^{*}\right|=2 \varepsilon n$ and $|U|+\left|N_{B_{6}}\left(V_{6}^{*} \backslash N_{B_{6}}(U)\right)\right| \leq\left|W_{6}\right|=2 \varepsilon n$, we have

$$
\begin{aligned}
\left|N_{B_{6}}(U)\right|+\left|V_{6}^{*} \backslash N_{B_{6}}(U)\right| & \geq|U|+\left|N_{B_{6}}\left(V_{6}^{*} \backslash N_{B_{6}}(U)\right)\right| \\
& >|U|+\left|V_{6}^{*} \backslash N_{B_{6}}(U)\right|
\end{aligned}
$$

that is, $\left|N_{B_{6}}(U)\right|>|U|$.

\section{RANDOM GRAPH IS GOOD.}

In order to show Lemma 7, we need to show that there exists a positive constant $C$ such that the random graph $G(n, p)$ with $p=C\left(\frac{\log n}{n}\right)^{1 / 2}$ a.a.s. satisfies Properties (P1) and (P2) in Definition 4 . Our proof of Properties (P1) and (P2) of $G(n, p)$ will be given in Sections 4.1 and 4.2 , respectively. In the proofs, we will use the following version of Chernoff's bound.

Lemma 12 (Chernoff's bound, Corollary 4.6 in [31]). Let $X_{i}$ be independent random variables such that $\operatorname{Pr}\left[X_{i}=1\right]=p_{i}$ and $\operatorname{Pr}\left[X_{i}=0\right]=1-p_{i}$, and let $X=\sum_{i=1}^{n} X_{i}$. For $0<\lambda<1$,

$$
\operatorname{Pr}[|X-\mathbb{E}(X)| \geq \lambda \mathbb{E}(X)] \leq 2 \exp \left(-\frac{\lambda^{2}}{3} \mathbb{E}(X)\right) .
$$

4.1. Property (P1). In order to show that $G(n, p)$ with $p=C\left(\frac{\log n}{n}\right)^{1 / 2}$ a.a.s. satisfies Property (P1), it suffices to show the following lemma.

Lemma 13. There exists a positive constant $C$ such that $G(n, p)$ with $p=C\left(\frac{\log n}{n}\right)^{1 / 2}$ a.a.s. satisfies the following: There exists a matching $\mathcal{M}$ with $|\mathcal{M}|=2 \varepsilon n$ in the subgraph of $G(n, p)$ induced on $V_{0}$ such that for all $U \subset V \backslash V(\mathcal{M})$ with $|U| \leq \frac{\delta n}{C^{2} \log n}$, we have

$$
\mid\{\{a, b\} \in \mathcal{M} \mid a \sim u, b \sim u \text { for some } u \in U\}\left|\geq \frac{C^{2} \log n}{16 n}\right| \mathcal{M}|| U \mid .
$$

Proof. Let $G_{1}=G\left(n-6 \varepsilon n, \frac{2 \log n}{n}\right)$ on the vertex set $V_{0}$ and $G_{2}=G(n, p / 2)$ on the vertex set $V$. It is easy to see that $G(n, p)$ on $V$ stochastically contains $G_{1} \cup G_{2}$. Hence, it is enough to show that $G_{1} \cup G_{2}$ a.a.s. contains a matching $\mathcal{M}$ described in Lemma 13 .

The result of Erdös and Rényi [23] implies that $G_{1}$ a.a.s. contains a matching in $V_{0}$ covering all but at most one vertex. Hence, $G_{1}$ on $V_{0}$ a.a.s. contains a matching of size $2 \varepsilon n$. Take such a matching $\mathcal{M}$ in $G_{1}$. 
Let

$$
X(U):=\mid\left\{e \in \mathcal{M} \mid e \subset N_{G_{2}}(u) \text { for some } u \in U\right\} \mid .
$$

Notice that $X(U)$ is the sum of independent and identically distributed (i.i.d.) random variables $X_{e}, e \in \mathcal{M}$, where

$$
X_{e}= \begin{cases}1 & \text { if } e \subset N_{G_{2}}(u) \text { for some } u \in U \\ 0 & \text { otherwise. }\end{cases}
$$

Since $|U| \leq \frac{\delta n}{C^{2} \log n}$, we have that for each $e \in \mathcal{M}$,

$$
\begin{aligned}
\operatorname{Pr}\left[X_{e}=0\right] & =\left(1-\left(\frac{p}{2}\right)^{2}\right)^{|U|} \leq 1-\frac{|U| p^{2}}{4}+\frac{|U|^{2} p^{4}}{32} \\
& \leq 1-\left(1-\frac{\delta}{8}\right) \frac{|U| p^{2}}{4} \leq 1-\frac{|U| p^{2}}{8}
\end{aligned}
$$

or equivalently, $\operatorname{Pr}\left[X_{e}=1\right] \geq \frac{1}{8}|U| p^{2}$. Hence,

$$
\mathbb{E}(X(U)) \geq \frac{p^{2}}{8}|\mathcal{M}||U|=\frac{C^{2} \log n}{8 n}|\mathcal{M}||U| .
$$

Chernoff's bound (Lemma 12 yields that

$$
\begin{aligned}
\operatorname{Pr}\left[X(U)<\frac{C^{2} \log n}{16 n}|\mathcal{M}||U|\right] & \leq \operatorname{Pr}\left[|X(U)-\mathbb{E}(X(U))| \geq \frac{1}{2} \mathbb{E}(X(U))\right] \\
& \leq 2 \exp \left(-0.01 p^{2}|\mathcal{M}||U|\right) \\
& =2 \exp \left(-0.01 \cdot 2 \varepsilon C^{2}|U| \log n\right) \\
& \leq 2 \exp (-3|U| \log n) \leq \frac{2}{n^{3|U|}}
\end{aligned}
$$

Therefore, we infer

$$
\begin{aligned}
\operatorname{Pr}[\exists U & \left.\in V \backslash V(\mathcal{M}) \text { with }|U| \leq \frac{\delta n}{C^{2} \log n} \text { such that } X(U)<\frac{C^{2} \log n}{16 n}|\mathcal{M}||U|\right] \\
& \leq \sum_{\ell=1}^{n} n^{\ell} \frac{2}{n^{3 \ell}} \leq n \cdot \frac{2}{n^{2}}=o(1),
\end{aligned}
$$

which completes the proof of Lemma 13 .

4.2. Property (P2). We now show that $G(n, p)$ with $p=C\left(\frac{\log n}{n}\right)^{1 / 2}$ a.a.s. satisfies Property (P2). First, recall the following definition which was given just before Definition 4 F For a graph $G$ on $V$ and $k=1$ or 2 , let $U \subset V$ and $\mathcal{L}$ be a collection of pairwise disjoint $k$-subsets of $V \backslash U$. We consider a bipartite graph $B(\mathcal{L}, U)$ between $\mathcal{L}$ and $U$, in which $L \in \mathcal{L}$ and $u \in U$ are adjacent if and only if $L \subset N_{G}(u)$.

In order to show that $G(n, p)$ with $p=C\left(\frac{\log n}{n}\right)^{1 / 2}$ a.a.s. satisfies Property (P2), it suffices to show the following lemma.

Lemma 14. There exists a positive constant $C$ such that $G(n, p)$ with $p=C\left(\frac{\log n}{n}\right)^{1 / 2}$ a.a.s. satisfies the following: Let $k=1$ or 2 , and $\mathcal{L}$ be a collection of pairwise disjoint $k$-subsets of $V$. 
(a) If $|\mathcal{L}| \leq \frac{\delta}{C^{k}}\left(\frac{n}{\log n}\right)^{k / 2}$, then, for $V_{i}$ with $V_{i} \cap\left(\bigcup_{L \in \mathcal{L}} L\right)=\emptyset, i=1, \ldots, 6$, we have that

$$
\left|N_{B\left(\mathcal{L}, V_{i}\right)}(\mathcal{L})\right| \geq(1-\delta) C^{k}\left(\frac{\log n}{n}\right)^{k / 2}|\mathcal{L}|\left|V_{i}\right|
$$

(b) If $|\mathcal{L}| \geq \frac{\log n}{C^{k-1}}\left(\frac{n}{\log n}\right)^{k / 2}$, then, for all $U$ with $|U| \geq \frac{\log n}{C^{k-1}}\left(\frac{n}{\log n}\right)^{k / 2}$ and $U \cap\left(\bigcup_{L \in \mathcal{L}} L\right)=\emptyset$, the graph $B(\mathcal{L}, U)$ has at least one edge.

Proof. For a proof of (a) of Lemma 14, we observe that $X\left(\mathcal{L}, V_{i}\right):=\left|N_{B\left(\mathcal{L}, V_{i}\right)}(\mathcal{L})\right|$ is the sum of i.i.d. random variables $X_{v}, v \in V_{i}$, where

$$
X_{v}= \begin{cases}1 & \text { if } L \subset N_{G}(v) \text { for some } L \in \mathcal{L} \\ 0 & \text { otherwise. }\end{cases}
$$

Since $|L|=k$ for all $L \in \mathcal{L}$ and $p^{k}|\mathcal{L}| \leq \delta=0.01$, we have

$$
\mathbb{E}\left(X\left(\mathcal{L}, V_{i}\right)\right)=\left|V_{i}\right|\left(1-\left(1-p^{k}\right)^{|\mathcal{L}|}\right) \geq(1-\delta / 2) p^{k}|\mathcal{L}|\left|V_{i}\right|=(1-\delta / 2) C^{k}\left(\frac{\log n}{n}\right)^{k / 2}|\mathcal{L}|\left|V_{i}\right| .
$$

Chernoff's bound (Lemma 12 implies that

$$
\begin{aligned}
\operatorname{Pr}\left[X\left(\mathcal{L}, V_{i}\right)<(1-\delta) C^{k}\left(\frac{\log n}{n}\right)^{k / 2}|\mathcal{L}|\left|V_{i}\right|\right] & \leq \operatorname{Pr}\left[\left|X\left(\mathcal{L}, V_{i}\right)-\mathbb{E}\left(X\left(\mathcal{L}, V_{i}\right)\right)\right| \geq \frac{\delta}{2} \mathbb{E}\left(X\left(\mathcal{L}, V_{i}\right)\right)\right] \\
& \leq 2 \exp \left(-\frac{\delta^{2}}{12} \mathbb{E}\left(X\left(\mathcal{L}, V_{i}\right)\right)\right),
\end{aligned}
$$

and by $p^{k}\left|V_{i}\right| \geq p^{2} \varepsilon n=C^{2} \varepsilon \log n$,

$$
\operatorname{Pr}\left[X\left(\mathcal{L}, V_{i}\right)<(1-\delta) C^{k}\left(\frac{\log n}{n}\right)^{k / 2}|\mathcal{L}|\left|V_{i}\right|\right] \leq 2 \exp (-3|\mathcal{L}| \log n)=\frac{2}{n^{3|\mathcal{L}|}} .
$$

Therefore, we infer that

$$
\begin{gathered}
\operatorname{Pr}\left[\exists V_{i}, \mathcal{L} \text { with } 1 \leq|\mathcal{L}| \leq \frac{\delta}{C^{k}}\left(\frac{n}{\log n}\right)^{k / 2} \text { such that } X\left(\mathcal{L}, V_{i}\right)<(1-\delta) C^{k}\left(\frac{\log n}{n}\right)^{k / 2}|\mathcal{L}|\left|V_{i}\right|\right] \\
\leq 6 \sum_{\ell=1}^{n} n^{\ell} \frac{2}{n^{3 \ell}} \leq 6 n \frac{2}{n^{2}}=o(1),
\end{gathered}
$$

which completes the proof of (a) of Lemma 14.

For a proof of (b) of Lemma 14 , we observe that the number $Y(\mathcal{L}, U)$ of edges in $B(\mathcal{L}, U)$ is the sum of i.i.d. random variables $Y_{L, u}$ for $L \in \mathcal{L}$ and $u \in U$, where

$$
Y_{L, u}= \begin{cases}1 & \text { if } L \subset N_{G}(u) \\ 0 & \text { otherwise. }\end{cases}
$$

Since $|L|=k$ for all $L \in \mathcal{L}$, we have $\mathbb{E}(Y(\mathcal{L}, U))=p^{k}|\mathcal{L}||U|$. Chernoff's bound (Lemma 12 yields that

$$
\begin{aligned}
\operatorname{Pr}[Y(\mathcal{L}, U)=0] & \leq \operatorname{Pr}\left[|Y(\mathcal{L}, U)-\mathbb{E}(Y(\mathcal{L}, U))| \geq \frac{1}{2} \mathbb{E}(Y(\mathcal{L}, U))\right] \\
& \leq 2 \exp \left(-\frac{1}{12} \mathbb{E}(Y(\mathcal{L}, U))\right) \leq 2 \exp \left(-\frac{1}{12} p^{k}|\mathcal{L}||U|\right)
\end{aligned}
$$


For $\ell \geq \frac{\log n}{C^{k-1}}\left(\frac{n}{\log n}\right)^{k / 2}$ and $r \geq \frac{\log n}{C^{k-1}}\left(\frac{n}{\log n}\right)^{k / 2}$, the number of $\mathcal{L}$ with $|\mathcal{L}|=\ell$ is at most $\left(\begin{array}{l}n \\ k\end{array}\right)^{\ell} \leq n^{k \ell}$ and the number of $U$ with $|U|=r$ is at most $\left(\begin{array}{l}n \\ r\end{array}\right)$, and we have

$$
\begin{aligned}
& \operatorname{Pr}[\exists \mathcal{L}, U \text { with }|\mathcal{L}|=\ell,|U|=r \text { such that } Y(\mathcal{L}, U)=0] \\
& \quad \leq n^{k \ell} n^{r} \cdot 2 \exp \left(-\frac{1}{12} p^{k} \ell r\right) \leq 2 \exp \left((k \ell+r) \log n-\frac{1}{12} p^{k} \ell r\right) .
\end{aligned}
$$

Since $p^{k} \ell=C^{k}\left(\frac{\log n}{n}\right)^{k / 2} \ell \geq C \log n$ and $p^{k} r=C^{k}\left(\frac{\log n}{n}\right)^{k / 2} r \geq C \log n$, we have that

$$
(k \ell+r) \log n \leq 0.01 C(\ell+r) \log n \leq 0.01\left(p^{k} \ell r+p^{k} \ell r\right) \leq 0.02 p^{k} \ell r
$$

and hence,

$n^{k \ell} n^{r} \cdot 2 \exp \left(-\frac{1}{12} p^{k} \ell r\right) \leq 2 \exp \left(-\frac{1}{24} p^{k} \ell r\right) \leq 2 \exp \left(-\frac{C^{2-k}}{24}\left(\frac{n}{\log n}\right)^{k / 2}(\log n)^{2}\right) \leq 2 \exp \left(-n^{1 / 2}\right)$.

Therefore, we infer that

$$
\begin{gathered}
\operatorname{Pr}\left[\exists \mathcal{L}, U \text { with }|\mathcal{L}|,|U| \geq \frac{\log n}{C^{k-1}}\left(\frac{n}{\log n}\right)^{k / 2} \text { such that } Y(\mathcal{L}, U)=0\right] \\
\leq \frac{n}{k} \cdot n \cdot 2 \exp \left(-n^{1 / 2}\right)=o(1),
\end{gathered}
$$

which completes the proof of (b) of Lemma 14 .

\section{CONCluding Remarks}

One may ask about how the approach of this paper can be used for the case that $d \geq 3$. We believe that our approach for finding a suitable matching given in Lemma 13 can be also applied in order to find a suitable family of vertex disjoint $d$-cliques when $d \geq 3$ and $p \geq C\left(\frac{\log n}{n}\right)^{1 / d}$. This approach together with an embedding algorithm modified from the algorithm in Dellamonica, Kohayakawa, Rödl and Ruciński [17, 18] may provide a simpler proof of Theorem 2.

As a further research direction, we are interested in resolving the following problem.

Problem 15. For an integer $d \geq 2$, determine the largest constant $a=a(d)$ with $0 \leq a \leq 1$ such that if $p \geq n^{-a+o(1)}$, then $G(n, p)$ is a.a.s. $\mathcal{F}_{n}(d)$-universal.

An easy observation mentioned in the introduction gives an upper bound $\frac{2}{d+1}$ for $a$. The current best lower bound is $\frac{1}{d}$ based on the result in Dellamonica, Kohayakawa, Rödl and Ruciński [17, 18] and this paper.

Acknowledgement. The second author thanks D. Dellamonica, Y. Kohayakawa, V. Rödl, and A. Rucinski for their helpful comments.

\section{REFERENCES}

[1] N. Alon and V. Asodi, Sparse universal graphs, J. Comput. Appl. Math. 142 (2002), no. 1, 1-11, Probabilistic methods in combinatorics and combinatorial optimization.

[2] N. Alon and M. Capalbo, Sparse universal graphs for bounded-degree graphs, Random Structures Algorithms 31 (2007), no. 2, 123-133.

[3] _ Optimal universal graphs with deterministic embedding, Proceedings of the Nineteenth Annual ACMSIAM Symposium on Discrete Algorithms (New York), ACM, 2008, pp. 373-378. 
[4] N. Alon, M. Capalbo, Y. Kohayakawa, V. Rödl, A. Ruciński, and E. Szemerédi, Universality and tolerance (extended abstract), 41st Annual Symposium on Foundations of Computer Science (Redondo Beach, CA, 2000), IEEE Comput. Soc. Press, Los Alamitos, CA, 2000, pp. 14-21.

[5] _ Near-optimum universal graphs for graphs with bounded degrees (extended abstract), Approximation, randomization, and combinatorial optimization (Berkeley, CA, 2001), Lecture Notes in Comput. Sci., vol. 2129, Springer, Berlin, 2001, pp. 170-180.

[6] N. Alon, M. Krivelevich, and B. Sudakov, Embedding nearly-spanning bounded degree trees, Combinatorica 27 (2007), no. 6, 629-644.

[7] L. Babai, F. R. K. Chung, P. Erdős, R. L. Graham, and J. H. Spencer, On graphs which contain all sparse graphs, Theory and practice of combinatorics, North-Holland Math. Stud., vol. 60, North-Holland, Amsterdam, 1982, pp. 21-26.

[8] J. Balogh, B. Csaba, M. Pei, and W. Samotij, Large bounded degree trees in expanding graphs, Electron. J. Combin. 17 (2010), no. 1, Research Paper 6, 9.

[9] J. Balogh, B. Csaba, and W. Samotij, Local resilience of almost spanning trees in random graphs, Random Structures Algorithms 38 (2011), no. 1-2, 121-139.

[10] S. N. Bhatt, F. R. K. Chung, F. T. Leighton, and A. L. Rosenberg, Universal graphs for bounded-degree trees and planar graphs, SIAM J. Discrete Math. 2 (1989), no. 2, 145-155.

[11] B. Bollobás, Random graphs, second ed., Cambridge Studies in Advanced Mathematics, vol. 73, Cambridge University Press, Cambridge, 2001.

[12] M. R. Capalbo and S. R. Kosaraju, Small universal graphs, Annual ACM Symposium on Theory of Computing (Atlanta, GA, 1999), ACM, New York, 1999, pp. 741-749 (electronic).

[13] F. R. K. Chung and R. L. Graham, On graphs which contain all small trees, J. Combinatorial Theory Ser. B 24 (1978), no. 1, 14-23.

[14] On universal graphs, Second International Conference on Combinatorial Mathematics (New York, 1978), Ann. New York Acad. Sci., vol. 319, New York Acad. Sci., New York, 1979, pp. 136-140.

[15] _ On universal graphs for spanning trees, J. London Math. Soc. (2) 27 (1983), no. 2, $203-211$.

[16] F. R. K. Chung, R. L. Graham, and N. Pippenger, On graphs which contain all small trees. II, Combinatorics (Proc. Fifth Hungarian Colloq., Keszthely, 1976), Vol. I, North-Holland, Amsterdam, 1978, pp. 213-223. Colloq. Math. Soc. János Bolyai, 18.

[17] D. Dellamonica, K. Kohayakawa, V. Rödl, and A. Ruciński, An improved upper bound on the density of universal random graphs, Submitted.

[18] D. Dellamonica, K. Kohayakawa, and A. Ruciński, An improved upper bound on the density of universal random graphs (extended abstract), Latin American Theoretical Computer Science Symposium, LATIN, 2012, pp. 231242 .

[19] D. Dellamonica, Jr. and Y. Kohayakawa, An algorithmic Friedman-Pippenger theorem on tree embeddings and applications, Electron. J. Combin. 15 (2008), no. 1, Research Paper 127, 14.

[20] D. Dellamonica, Jr., Y. Kohayakawa, V. Rödl, and A. Ruciński, Universality of random graphs, Proceedings of the Nineteenth Annual ACM-SIAM Symposium on Discrete Algorithms (New York), ACM, 2008, pp. $782-788$.

[21] _ Universality of random graphs, SIAM J. Discrete Math. 26 (2012), no. 1, 353-374.

[22] P. Erdös, Some remarks on the theory of graphs, Bull. Amer. Math. Soc. 53 (1947), 292-294.

[23] P. Erdős and A. Rényi, On the existence of a factor of degree one of a connected random graph, Acta Math. Acad. Sci. Hungar. 17 (1966), 359-368.

[24] J. Friedman and N. Pippenger, Expanding graphs contain all small trees, Combinatorica 7 (1987), no. 1, 71-76.

[25] A. Hajnal and E. Szemerédi, Proof of a conjecture of P. Erdös, Combinatorial theory and its applications, II (Proc. Colloq., Balatonfüred, 1969), North-Holland, Amsterdam, 1970, pp. 601-623.

[26] S. Janson, T. Łuczak, and A. Rucinski, Random graphs, Wiley-Interscience Series in Discrete Mathematics and Optimization, Wiley-Interscience, New York, 2000. 
[27] D. Johannsen, M. Krivelevich, and W. Samotij, Expanders are universal for the class of all spanning trees, Combin. Probab. Comput. 22 (2013), no. 2, 253-281.

[28] A. Johansson, J. Kahn, and V. Vu, Factors in random graphs, Random Structures Algorithms 33 (2008), no. 1, $1-28$.

[29] J. Komlós and E. Szemerédi, Limit distribution for the existence of Hamiltonian cycles in a random graph, Discrete Math. 43 (1983), no. 1, 55-63.

[30] A. Korshunov, A solution of a problem of P. Erdös and A. Rényi about hamilton cycles in non-oriented graphs, Metody Diskr. Anal. Teoriy Upr. Syst., Sb. Trudov Novosibirsk. 31 (1977), 17-56 (in Russian).

[31] M. Mitzenmacher and E. Upfal, Probability and computing, Cambridge University Press, Cambridge, 2005, Randomized algorithms and probabilistic analysis.

[32] V. Rödl, A note on universal graphs, Ars Combin. 11 (1981), 225-229.

School of Computational Sciences, Korea Institute for Advanced Study (KIAS), Seoul, South Korea (J. H. Kim)

E-mail address: jhkim@kias.re.kr

Department of Mathematical Sciences, Korea Advanced Institute of Science and Technology (KAIST), Daejeon, South Korea (S. J. Lee)

E-mail address: sjlee242@gmail.com 